\title{
Stochastic molecular modeling the transport coefficients of rarefied gas and gas nanosuspensions
}

\author{
V. Ya. Rudyak ${ }^{1,2}$, E. V. Lezhnev ${ }^{1}$ \\ ${ }^{1}$ Novosibirsk State University of Architecture and Civil Engineering, Leningradskaya, 113, Novosibirsk, Russia \\ ${ }^{2}$ Novosibirsk State University, Pirogova, 1, Novosibirsk, Russia \\ valery.rudyak@mail.ru, lionlev@yandex.ru
}

PACS 02.70.Ns, 02.70.Tt, 47.55.-n, 51.20.+d, 47.57.J

DOI 10.17586/2220-8054-2020-11-3-285-293

\begin{abstract}
The subject of this paper is the stochastic molecular modelling of the transport coefficients for rarefied gases and gas nanosuspensions. The proposed method is an alternative one to the molecular dynamics method. However, unlike the latter, the phase trajectories of the molecular system are simulated stochastically. Adequate integral characteristics of the studied system are obtained by averaging the calculated data over independent phase trajectories. The efficiency of the proposed algorithm is demonstrated by calculation of the diffusion and viscosity coefficients of several noble and polyatomic gases and rarefied gas nanosuspensions. The modeling accuracy increases when a greater number of molecules and phase trajectories are employed.
\end{abstract}

Keywords: diffusion, gas nanosuspension, rarefied gas, stochastic molecular simulation; transport processes, transport coefficients, viscosity.

Received: 18 April 2020

Revised: 22 April 2020

\section{Introduction}

Transport processes determine characteristics of all natural and industrial flows of liquids and gases. The description of these processes requires knowledge of the corresponding transport coefficients: diffusivity, viscosity, thermal conductivity, etc. Of course, there are fairly reliable experimental methods for measuring them, however, the necessity of their theoretical calculations or simulations is also understandable. In rarefied gases, this problem is solved using kinetic theory of gases (see, for example, [1]). However, calculation of these coefficients is generally a non-trivial task. It is necessary to solve complex integral equations. The solution of these equations is a rather cumbersome and complicated procedure [2,3]. In addition, so-called $\Omega$-integrals have to be calculated. There are the sufficiently detailed tables of the values of these integrals for the Lennard-Jones potential only.

Molecular modeling is single alternative of the experimental determination of the transport coefficients of liquid and gases. Practically, the molecular dynamics (MD) method is the only consistent method for such modeling. However this method is not applicable to simulation of the rarefied gases because the corresponding simulation cell should have a characteristic linear dimension much larger than the mean free path of molecules and it is therefore necessary to use a huge number of molecules. On the other hand, systematic studies of the MD method have shown that it does not provide true phase trajectories of molecular systems (see [4-6]). Adequate results of MD simulations can be obtained only by averaging the corresponding dynamical variables over an ensemble of independent phase trajectories.

In our papers [7,8], a stochastic algorithm to model the transport coefficients of the rarefied gases has been developed. In this algorithm the phase trajectories of the molecular system are simulated stochastically. In the present paper, the given algorithm is generalized for modeling the transport coefficients of rarefied polyatomic gases and rarefied gas nanosuspensions.

\section{Simulation of the molecules phase trajectories}

In rarefied gases, only binary collisions of molecules take place and the molecules themselves are material points. The spatiotemporal scales of the order of the time of the molecules interaction and their size are indistinguishable. This implies that the intermolecular interactions do not contribute to the transport coefficients and the equation of state. Such contributions appear only in the next approximation for density (for moderately dense gas) $[9,10]$. For this reason, the transport coefficients of a rarefied gas are only functions of the velocities of molecules [1-3,10]. Therefore, to model the transport coefficients of the rarefied gas, it is sufficient to study its evolution in the space of velocities.

Let us consider the system of gas molecules with numerical density $n$. At the initial time, the velocities of the molecules are distributed according to the Maxwell distribution function at a given temperature $T$. Simulation of the gas dynamics starts with generating a list of velocities of the molecules at the initial time $t$. Thus, at time $t$, the 
molecules have velocities $\left(\mathbf{v}_{1}, \mathbf{v}_{2}, \ldots \mathbf{v}_{N}\right)$. It is necessary to obtain the values of the molecules velocities at successive times in the simulation interval $\left(t, t_{s}\right)$.

The dynamics of the molecules are determined by their interactions. Below these interactions are described by the Lennard-Jones potential:

$$
U(r)=4 \varepsilon\left[\left(\frac{\sigma}{r}\right)^{12}-\left(\frac{\sigma}{r}\right)^{6}\right] .
$$

Here, $\sigma$ is the effective radius of the molecule and $\varepsilon$ is the maximum value of the attraction energy. In this paper, the gas mixtures are considered; therefore, in addition to the interaction of their molecules, which are described by the potential parameters $(1), \sigma_{i i}, \varepsilon_{i i}(i=1,2)$, it is necessary to determine the parameters of the cross-interactions of molecules of type $i$ with molecules of type $j: \sigma_{i j}, \varepsilon_{i j}$. These parameters were determined using the simplest combination relations: $\varepsilon_{i j}=\sqrt{\varepsilon_{i i} \varepsilon_{j j}}, \sigma_{i j}=\left(\sigma_{i i}+\sigma_{j j}\right) / 2$. Since the potentials have an infinite radius of action, they should be truncated in the course of simulation. The truncation radii are equal to $2.5 \sigma$ for potential (1).

As the first step of the algorithm constructing the time interval $\tau_{1}=\sigma / v_{\max }$ is determined. Here, $v_{\max }$ is the maximum magnitude of the velocity of the molecules in the system at the given time. The generation of a list of the molecules velocities at the time $\left(t+\tau_{1}\right)$ starts with a consideration of molecule 1 . For a one-component system, to determine whether a molecule 1 collides with any other molecule during time $\tau_{1}$, a random number $P$ uniformly distributed in the interval $(0,1)$ is generated. If, in time $\tau_{1}$, it is less than the average probability of molecule collision $P_{\tau_{1}}=4 \tau_{1} n r_{c}^{2} \sqrt{\pi k T / m}$ (see [1]), then molecule with which molecule 1 collides is randomly selected from the remaining $(N-1)$ molecules (here $r_{c}$ is the potential cut-off radius of the potential (1), $m$ is the mass of molecule and $k$ is the Boltzmann constant). As a result, the velocities of the colliding molecules change according to the laws of elastic collision:

$$
\mathbf{v}_{1}^{\prime}=\mathbf{v}_{1}+\left(\mathbf{v}_{1 j} \cdot \mathbf{e}_{1 j}\right) \mathbf{e}_{1 j}, \quad \mathbf{v}_{j}^{\prime}=\mathbf{v}_{j}-\left(\mathbf{v}_{1 j} \cdot \mathbf{e}_{1 j}\right) \mathbf{e}_{1 j},
$$

where $\mathbf{v}_{1 j}=\left(\mathbf{v}_{1}-\mathbf{v}_{j}\right)$ is the vector of relative velocity and $\mathbf{e}_{1 j}$ is the unit vector from center of molecule $j$ to molecule 1. The actual mechanisms of the molecules interaction is described in detailed in paper [8].

If the generated number $P$ is greater than the average probability of collision, molecule 1 does not collide in this time interval and its velocity does not change. If it collides, the velocities of molecules 1 and $j$ in the initial list change. The remaining molecules are treated similarly. As a result, a new list of the velocities of the molecules $\left(\mathbf{v}_{1}^{\prime}, \mathbf{v}_{2}^{\prime}, \ldots \mathbf{v}^{\prime}{ }_{N}\right)$ is generated.

After the generation of the list for the time $\left(t+\tau_{1}\right)$, the next time interval is selected as $\tau_{2}=\sigma / v_{\max }$, where $v_{\max }$ is the maximum magnitude of the velocity of the molecules of the system at the time $\left(t+\tau_{1}\right)$ and the procedure is repeated. This procedure is repeated until the given simulation time $t_{s}$ equal to $t_{s}=\tau_{1}+\tau_{2}+\ldots+\tau_{k}$ is reached. The calculation result is the full set of the velocities of all the molecules of the simulated system at successive times.

For mixer of the gases this algorithm is slightly modified. Consider, for example, the binary mixer. In this case, the time step $\tau_{1}$ is determined by the following relation $\tau_{1}=\sigma_{\min } / v_{\max }$, where $\sigma_{\min }=\min \left(\sigma_{11}, \sigma_{22}\right)$. Now the molecule of sort 1 can collide with molecule of the same type or molecule of sort 2 . Let us consider for definiteness, a particle of the first sort (for the second the formulas are similar). In this case, the collision probability is equal to:

$$
P_{\tau_{1}}=4 \tau_{1} n_{1} r_{c 11}^{2} \sqrt{\frac{\pi k T}{m_{1}}}+2 \tau_{1} n_{2} r_{c 22}^{2} \sqrt{\frac{2 \pi k T}{x_{1} m_{2}}}=P_{11}+P_{12} .
$$

Here, $r_{c i i}$ is the cut-off intermolecular radius of the potential (1) for molecules of sort $i$ and $x_{1}$ is the molar fraction of the molecules of the first sort.

So, if the generated number $P$ is smaller than $P_{\tau_{1}}$, the collision will occur and for molecule 1 of sort 1 , randomly from the $N-1$ remaining molecules, the particle $j$ is selected with which this collision will be realized. Moreover, if $P<P_{11}$, a molecule of the first sort is selected, and if $P_{11}<P$ then it is of the second sort molecule. In addition, the velocities of the molecules after collision are determined by the following relation (compare with equation (2)):

$$
\mathbf{v}_{1}^{\prime}=\mathbf{v}_{1}+2 M_{j}\left(\mathbf{v}_{1 j} \cdot \mathbf{e}_{1 j}\right) \mathbf{e}_{1 j}, \quad \mathbf{v}_{j}^{\prime}=\mathbf{v}_{j}-2 M_{1}\left(\mathbf{v}_{1 j} \cdot \mathbf{e}_{1 j}\right) \mathbf{e}_{1 j},
$$

where $M_{i}=m_{i} /\left(m_{1}+m_{j}\right)$.

\section{Diffusion and viscosity coefficients of rarefied gases}

The described algorithm permits one to model transport processes in rarefied gases. In particular, using this algorithm it may be used to calculate the transport coefficients. The transport coefficients of the molecular system considered are calculated using fluctuation-dissipation theorems, which relate transport coefficients to the evolution of the corresponding correlation functions. In the literature, these relations are called the Green-Kubo formulas [4, 10-12]. The identity of these formulas and relations of the kinetic theories [1-3] was studied and established in paper $[13,14]$. 
According to the fluctuation-dissipation theorem the binary diffusion coefficient is described by the following formulas [15]:

where

$$
D=\frac{1-x_{1}}{x_{1}} L_{11}+\frac{x_{1}}{1-x_{1}} L_{22}-L_{12}-L_{21}
$$

$$
\begin{gathered}
L_{\alpha \beta}=\frac{1}{3 N} \int_{0}^{\tau_{p}} d t \chi_{\alpha \beta}(0, \tau), \\
\chi_{\alpha \beta}(0, t)=\sum_{k}^{N_{\alpha}}\left[v_{k \alpha}(0)-v(0)\right] \cdot \sum_{l}^{N_{\beta}}\left[v_{l \beta}(0)-v(0)\right], \\
v(t)=\frac{x_{1}}{N_{1}} \sum_{k}^{N_{\alpha}} v_{k \alpha}(t)+\frac{1-x_{1}}{N_{2}} \sum_{l}^{N_{\beta}} v_{l \beta}(t) .
\end{gathered}
$$

Here, $t_{p}$ is so named plateau time of the calculation of the integral (3) [16].

In the formulas (3), the integrand defines the correlation functions of the molecular velocities, the time integrals of which give the value of the diffusion coefficient. The autocorrelation velocity function (ACVF) of the rarefied gas should decay exponentially [4]. As an example in Fig. 1 (left), the evolution of the ACVF $\chi_{11}$ for the $\mathrm{Kr}$-Ar mixture is given. Here, $t^{\prime}=t / \tau$, where $\tau$ is the mean free path time of Kr molecules. In actuality, ACVF decays exponentially. Therefore, the plateau value of the diffusion coefficient is reached within 10-15 mean free path times (see Fig. 1 right).
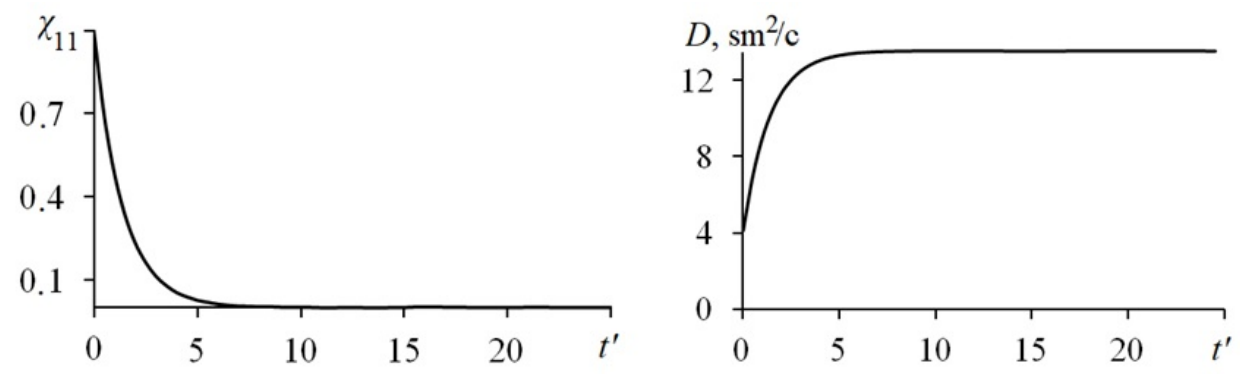

FIG. 1. The evolution of ACVF (left) and diffusion coefficient of Kr-Ar mixer (right)

The accuracy of the simulation was evaluated by comparison with experimental data. Firstly, the diffusion coefficients for mixtures of rarefied noble gases $\mathrm{Kr}-\mathrm{Ar}$, Xe-Ar, $\mathrm{Xe}-\mathrm{Kr}$ at atmospheric pressure and temperature of $295 \mathrm{~K}$ were analyzed. The following intermolecular potential parameters (see (1)) were used: $\sigma=0.311 \mathrm{~nm}, \varepsilon / k=116 \mathrm{~K}$ for argon, $\sigma=0.351 \mathrm{~nm}, \varepsilon / k=190 \mathrm{~K}$ for krypton and $\sigma=0.386 \mathrm{~nm}, \varepsilon / k=190 \mathrm{~K}$ for Xenon [2]. Calculation of each phase trajectory was performed for 50 mean free paths time. The diffusion coefficient $D$ was calculated by averaging over 1000 independent phase trajectories. 3500 molecules were used in the calculations. Comparison of simulated and experimental data for the three mixtures is presented in Table 1. Here the second line corresponds to the experimental data $D_{e}[17]$ and the relative error of the simulation is given in the last line.

TABLE 1. Comparison of simulated and experimental data of the diffusion coefficients

\begin{tabular}{|c|c|c|c|}
\hline$T$ & $\mathrm{Kr}-\mathrm{Ar}$ & $\mathrm{Xe}-\mathrm{Ar}$ & $\mathrm{Xe}-\mathrm{Kr}$ \\
\hline$D_{e}, \mathrm{sm}^{2} / \mathrm{s}$ & 13.58 & 11.1 & 7.43 \\
\hline$D, \mathrm{sm}^{2} / \mathrm{s}$ & 13.22 & 11.42 & 7.19 \\
\hline$\Delta, \%$ & 2.63 & 2.88 & 3.28 \\
\hline
\end{tabular}

The simulation error of the diffusion coefficients was about 3\% (see Table 1). It should be noted that the accuracy of measuring the diffusion coefficient also usually lies in the range of 1-3\%. So the accuracy of modeling, even with the use of this relatively small number of molecules, is quite acceptable. However, the accuracy of the simulation using the presented algorithm increases with the number of molecules used and/or the number of phase trajectories used for averaging. It will be discussed later.

One of the main problems of the kinetic theory of gases is the modeling transport processes of polyatomic gases. Different approaches are used here, including the use of various semi-classical or quantum methods (see, for example, $[2,3,18,19])$. Nevertheless, even in the simplest case of a single-component gas, the calculation of the transport coefficients of a rarefied polyatomic gas has not completely solved. Therefore, it would be of utility to understand 
the applicability of the algorithm described above for modeling transport coefficients of polyatomic gases. Below this problem is analyzed on example of modeling the viscosity coefficient both monatomic ( $\mathrm{Ar}, \mathrm{Kr}, \mathrm{Ne}, \mathrm{Xe}$ ) and polyatomic gases $\left(\mathrm{CH}_{4}, \mathrm{CO}, \mathrm{CO}_{2}, \mathrm{O}_{2}\right)$.

The calculation of the viscosity coefficient, as using MD method, is based on the fluctuation-dissipation theorem, which has the form for the viscosity coefficient [4, 10-12]:

$$
\eta=\frac{1}{3 V K T} \int_{0}^{t_{p}} d t \chi_{\eta}(0, t)=\frac{1}{3 V K T} \int_{0}^{t_{p}} d t\left\langle\sigma_{x y}(0) \sigma_{x y}(t)\right\rangle,
$$

where $V$ is the volume of the system. The components of the stress tensor for a rarefied gas are calculated as:

$$
\sigma_{x y}(0, t)=\frac{1}{N l} \sum_{j}^{l} \sum_{k}^{N} m\left[v_{x k}(j \Delta t) v_{y k}(t+j \Delta t)\right]
$$

Here $\Delta \mathrm{t}$ is the time interval of the calculation of the velocities.

To simulate the viscosity of the gases the following intermolecular potential parameters were used: $\sigma=0.382 \mathrm{~nm}$, $\varepsilon / k=137 \mathrm{~K}$ for $\mathrm{CH}_{4}, \sigma=0.359 \mathrm{~nm}, \varepsilon / k=110 \mathrm{~K}$ for $\mathrm{CO}, \sigma=0.448 \mathrm{~nm}, \varepsilon / k=189 \mathrm{~K}$ for $\mathrm{CO}_{2}, \sigma=0.343 \mathrm{~nm}$, $\varepsilon / k=113 \mathrm{~K}$ for $\mathrm{O}_{2}, \sigma=0.178 \mathrm{~nm}, \varepsilon / k=35.7 \mathrm{~K}$ for Ne. The viscosity coefficients were calculated at a temperature of $273 \mathrm{~K}$ and atmospheric pressure. In all cases 3200 molecules were used in the calculations, and the obtained data were averaged over 1000 independent phase trajectories.

The correlation of the stress tensor $\chi_{n}(t)$ decays exponentially also. The obtained values of the viscosity coefficient are presented in Table 2 (second line). The corresponding experimental values of $\eta_{e}$ taken from the reference book [20] are also shown here. The relative error of the simulation is given in the last line again.

TABLE 2. Comparison of simulated and experimental data of the viscosity coefficients of the rarefied gases

\begin{tabular}{|c|c|c|c|c|c|c|c|c|}
\hline & $\mathrm{CH}_{4}$ & $\mathrm{CO}$ & $\mathrm{CO}_{2}$ & $\mathrm{O}_{2}$ & $\mathrm{Ar}$ & $\mathrm{Kr}$ & $\mathrm{Ne}$ & $\mathrm{Xe}$ \\
\hline$\eta \cdot 10^{6}(\mathrm{~Pa} \cdot \mathrm{s})$ & 10.48 & 15.95 & 13.0 & 18.91 & 22.25 & 25.87 & 32.28 & 23.78 \\
\hline$\eta_{e} \cdot 10^{6}(\mathrm{~Pa} \cdot \mathrm{s})$ & 10.37 & 16.6 & 13.8 & 19.3 & 22.7 & 25.5 & 31.7 & 23.3 \\
\hline$\Delta, \%$ & 1.06 & 3.94 & 5.84 & 2.02 & 1.97 & 1.46 & 1.84 & 2.05 \\
\hline
\end{tabular}

Comparison of simulation and experimental data shows that the simulation accuracy is within the accuracy of obtaining experimental data for monoatomic gases and oxygen (about 2\%). At the same time, the situation for polyatomic gases is more complicated. Good data are obtained for methane, whose molecules are spherically symmetric and for oxygen. On the other hand, for non-spherical molecules, of course, more adequate potentials should be applied. Although here, the accuracy of modeling the viscosity coefficient is not bad. In addition, it can also be increased by using a larger number of molecules (see the next section).

\section{Accuracy of the modeling}

The calculations presented in previous section showed that the proposed algorithm for modeling gas transport coefficients allows one, using a relatively small number of molecules, to obtain data comparable in accuracy with experimental results. However, the dependence of the simulation results on the molecules number used is a very important feature of the algorithm. The dependence of the accuracy of the number of particles was studied in modeling different transport coefficients. Below, the obtained results will be shown for calculating the diffusion and viscosity coefficients of a few rarefied gases. In all cases, the simulation results were compared with the corresponding experimental ones.

Let us consider the diffusion of Ar-Kr mixture. The number of the used molecules $N$ was varied from 850 to 6800. The obtained relative error ranges from $4.9 \%$ to $1.9 \%$. These data indicate that the accuracy of the simulation increases with an increasing number of molecules. One can show that the resulting relative errors $\Delta$ are well described by the dependence $\Delta \sim 1 / \sqrt{N}$.

A similar result is obtained when modelling the viscosity coefficient. As an example, the dependence of the accuracy of the number of particles was studied in modeling the viscosity coefficient of argon. The data obtained in simulations of $10,100,500,1600$, and 3200 molecules are analyzed. The obtained relative error is changed from $9.7 \%$ to $0.8 \%$ and again, $\Delta \sim 1 / \sqrt{N}$.

Another important factor determining the simulation's accuracy is the number of ensemble members over which the averaging is performed. The ensemble of the phase trajectories for averaging is a typical Gibbs ensemble characterized by different initial phase states of molecules with given mean values of macroscopic observables (temperature, 
pressure, density). To study the dependence of the simulation accuracy on the number of ensemble members $L$ (the number of independent phase trajectories) over which the averaging is performed, the diffusion of an Ar-Kr mixture is again considered. The parameter $L$ was changed from 125 to 1000 , the number of the molecules was the same and equal to 3200 . The relative accuracy of the diffusion coefficient changed from $5.8 \%$ to $2.6 \%$. These (and many other) test calculations have shown that the simulation's accuracy $\Delta_{s} \sim 1 / \sqrt{L}$. This estimate is expected, of course. However, it is clear that the value $\Delta_{s} \sim 10^{-2}$ does not mean that accuracy of the order of one percent is achieved. The accuracy also depends on the number of molecules in the computational cell.

Averaging the simulated observables over a sufficiently large number of the independent phase trajectories is connected in particular with the nonmonotonic damping of the two-time correlation functions of the molecular systems (see, for example, papers [21, 22], in which the quasi-periodic behavior of ACVF was predicted). In finite number molecule system the amplitude of these fluctuations may be rather large. Therefore, a comparatively large number of the used phase trajectories is required to achieve the high accuracy of the modelling.

On the other hand, one needs to repeat that the simulation accuracy, of course, depends on the number of molecules used. For a very small number of particles, sufficiently accurate results were not obtained. The reason in the rarefied gas is clear: in a rarefied gas, the collided molecules do not collide in future. The probability of such collisions is negligible, and they are a characteristic feature of the dynamics of dense gases $[9,10]$. On the other hand, in a system of a small number of molecules, modeling the rarefied gas during the time $\tau_{i}$ there are a significant number of repeated collisions involving the same molecules [23,24]. The simulation's accuracy can be substantially increased by eliminating such collisions. In the Bird method [25] of direct statistical Monte-Carlo method, this was done in paper [26]. Elimination of repeated collisions made it possible to achieve the same accuracy for ten particles as when using the standard method for one thousand particles. A similar procedure was realized in the algorithm we describe here. The procedure of eliminating repeated collisions is extremely simple. If, in the selection of the collision of the $i$-th molecule, it is to collide with molecule $k$, and this collision has already occurred in the given time interval, then a different pair for collision is selected. The example of the calculations of the viscosity coefficients of $\mathrm{CH}_{4}$ gas with (second line) and without (third line) repeated collisions are presented in Table 3. In all presented calculations the averaging of the data were fulfilled over thousand independent phase trajectories. The result is excellent, the acceptable accuracy is obtained when using only ten molecules.

TABLE 3. Comparison of simulated data of the viscosity coefficients for methane

\begin{tabular}{|c|c|c|c|c|}
\hline$N$ & 10 & 100 & 500 & 2500 \\
\hline$\Delta_{0}, \%$ & 11.06 & 5.89 & 4.83 & 4.24 \\
\hline$\Delta_{1}, \%$ & 2.18 & 1.76 & 1.43 & 1.27 \\
\hline
\end{tabular}

Using the described algorithm for eliminating repeated collisions it is possible to reduce by one or even several orders of magnitude the number of molecules used for modeling. Naturally, eliminating repeated collisions the simulation time may be considerably reduced. On the other hand, it is clear that the effectiveness of the procedure for excluding repeated collisions decreases with an increasing the number of molecules used in the simulation.

\section{Modeling the transport processes in rarefied gas nanosuspensions}

A gas nanosuspension is a two-phase medium in which the carrier medium is a rarefied gas and dispersed particles are nanoparticles having the size from 1 to $100 \mathrm{~nm}$. For considering gas nanosuspensions, the mean free path of the molecules is more than size of dispersed particles, as a rule. The key point for modeling the dynamics of gas nanosuspensions is the interaction potentials of the nanoparticles with molecules of the carrier gas and nanoparticles with each other. In MD modeling of transport processes of nanofluids, the nanoparticles are often simulated by the clusters of different size (see review [27] and references therein). But molecular cluster is not nanoparticle because in gas and liquid it may change (and change!) its size and shape. In addition, the atoms of such cluster can be replaced by the base fluid molecules. On the other hand, in real physical experiment we have stable nanoparticles, these particles have constant shape and size. In order to simulate the transport processes in such nanofluids, it is necessary to determine correctly the interaction potentials of nanoparticles with base fluid molecules and between each other. Such potentials were constructed early in papers [28,29]. Later the use of this potential was experimentally checked [30].

The interaction of molecules of the carrier medium with a nanoparticle was determined by the Rudyak-Krasnolutskii (RK) potential as follows [28,29]:

$$
\Psi(r)=\Psi_{9}(r)-\Psi_{3}(r)
$$




$$
\Psi_{i}(r)=C_{i}\left\{\left[(r-R)^{-i}-(r+R)^{-i}\right]\right\}-a_{i} r^{-1}\left[(r-R)^{-i+1}-(r+R)^{-i+1}\right],
$$

where $i=9,3, a_{9}=9 / 8, a_{3}=3 / 2, C_{9}=\left(4 \pi \varepsilon_{12} \sigma_{12}^{12}\right) / 45 V_{p}, C_{3}=\left(2 \eta \varepsilon_{12} \sigma_{12}^{6}\right) / 3 V_{p}$, and $V_{p}^{-1}=\rho_{p} / m_{p}$.

Here, $\rho_{p}$ is the density of the nanoparticle material, $m_{p}$ is the molecular mass of the substance of the nanoparticle, $R$ is the nanoparticle radius, $\sigma_{i j}$ and $\varepsilon_{i j}$ are the parameters of potential (1) of the interactions of a carried medium molecule with a nanoparticle atom (or molecule).

As the interaction potential for nanoparticles, we are using the potential [31], which has the following form for monodisperse particles:

$$
\begin{gathered}
U(r, R)=U_{7}(r, R)-U_{1}(r, R) \\
U_{7}(r)=\frac{\pi^{2} \tilde{\varepsilon} \tilde{\sigma}^{12}}{315 V_{p}^{2}}\left\{\frac{R^{2}}{r}\left[\frac{1}{(r-2 R)^{7}}+\frac{2}{R^{7}}+\frac{1}{(r+2 R)^{7}}\right]-\frac{R}{3 r}\left[\frac{1}{(r-2 R)^{6}}-\frac{1}{(r+2 R)^{6}}\right]-\right. \\
\left.\quad-\frac{1}{30 r}\left[\frac{1}{(r-2 R)^{5}}-\frac{2}{R^{5}}+\frac{1}{(r+2 R)^{5}}\right]\right\}, \\
U_{7}(r)=\frac{2 \pi^{2} \tilde{\varepsilon} \tilde{\sigma}^{6}}{3 V_{p}^{2}}\left[\ln \left(\frac{r^{2}-4 R^{2}}{r^{2}}\right)+2 R^{2}\left(\frac{1}{r^{2}-4 R^{2}}+\frac{1}{r^{2}}\right)\right],
\end{gathered}
$$

here $\tilde{\varepsilon}$ and $\tilde{\sigma}$ are the parameters of the Lennard-Jones interaction potential (1) of the nanoparticle atoms (molecules). Potentials (5) and (6) were constructed on the assumption that the interaction of carrier fluid molecules with nanoparticle atoms and nanoparticle atoms with each other is described by a potential of the form (1) with the parameters $\sigma_{12}, \varepsilon_{12}$ and $\tilde{\sigma}, \tilde{\varepsilon}$, respectively. Since these potentials have an infinite radius of action, they should be truncated in the course of simulation. The truncation radii are equal to $R+b \sigma_{12}$ for potentials (5) and (6). Factor $b$ was selected such that the force with which a nanoparticle acts on a molecule is equal to the force of interaction between two molecules over the truncation radius for potential (5). This factor depends on the nanoparticle material and the properties of carrier molecules.

In this paper, rarefied gas nanosuspensions are considered. It is a binary system. The algorithm to simulate such system is described in section 2. The result of the calculations is a complete set of velocities of all molecules and nanoparticles of the simulated system at successive times. Using this information, it is possible to calculate all the observable characteristics of the system studied. The transport coefficients studied here were calculated again using the fluctuation-dissipation theorems (3) and (4).

One of the goals of this paper was to study the possibilities of the proposed simulation algorithm for modeling the diffusion of nanoparticles in gas. Previously, the diffusion of $\mathrm{Cu}_{2} \mathrm{O}$ nanoparticles in nitrogen at atmospheric pressure and temperature $294.15 \mathrm{~K}$ was studied experimentally [30]. Therefore for testing the proposed algorithm, namely this diffusion was modeled. The following parameters of the intermolecular interaction potential were used: $\sigma=0.3798 \mathrm{~nm}$, $\varepsilon / k=71.4 \mathrm{~K}$ for $\mathrm{N}_{2}, \sigma=0.4124 \mathrm{~nm}, \varepsilon / k=2909 \mathrm{~K}$ for $\mathrm{Cu}_{2} \mathrm{O}$ [30]. The diameter of $\mathrm{Cu}_{2} \mathrm{O}$ particle was varied from 2.55 to $8.94 \mathrm{~nm}$. The number of molecules in the calculations varied from 450 to 20,000, depending on the size of the nanoparticle.

The comparison of simulation $D$ and experimental $D_{e}$ data of the diffusion coefficient of $\mathrm{Cu}_{2} \mathrm{O}$ particles is shown in Table 4. Here, the first column is the diameter of the nanoparticle and the fourth is the relative error of the simulation. In all cases, the simulation accuracy is quite high and practically does not depend on the size of the nanoparticles.

TABLE 4. Calculations of the diffusion coefficient of the nanoparticles

\begin{tabular}{|c|c|c|c|}
\hline$R, \mathrm{~nm}$ & $D, \mathrm{~m}^{2} / \mathrm{s} \cdot 10^{-7}$ & $D_{e}, \mathrm{~m}^{2} / \mathrm{s} \cdot 10^{-7}$ & $\Delta, \%$ \\
\hline 2.55 & 5.156 & 5.063 & 1.84 \\
\hline 2.94 & 4.046 & 4.102 & 1.37 \\
\hline 3.33 & 3.386 & 3.418 & 0.95 \\
\hline 4.51 & 2.158 & 2.118 & 1.87 \\
\hline 4.79 & 1.968 & 1.932 & 1.88 \\
\hline 5.35 & 1.632 & 1.601 & 1.95 \\
\hline 6.33 & 1.236 & 1.215 & 1.71 \\
\hline 7.54 & 9.196 & 9.044 & 1.68 \\
\hline 8.94 & 6.625 & 6.694 & 1.03 \\
\hline
\end{tabular}

The rarefied gas nanosuspension is a special case of a nanofluid. Nanofluids with spherical nanoparticles and carbon nanotubes have already been successfully used or are proposed for use in various applications (see for example 
[32-35]). The model of the viscosity of coarse dispersed fluid was developed by Einstein [36] and then generalized by many authors (see [37-40] and references therein). It was established that viscosity of coarse dispersed fluid is always larger than the viscosity of based fluid and this increase depends on volume concentration of the dispersed particles. In nanofluids this tendency is conserved but their viscosity depends not only on the particles concentration but also on size and material of nanoparticles [41,42]. However, in papers [43,44], it is shown using the kinetic theory that the viscosity of the rarefied gas nanosuspensions can be either greater or less than the viscosity of the carrier gas. One of the tasks of this paper is to test the possibility of describing this effect using the proposed stochastic molecular modeling algorithm. The viscosity of the two different gas nanosuspensions $\left(\mathrm{Zn}-\mathrm{Ne} \mathrm{U}-\mathrm{H}_{2}\right)$ are studied. The following parameters of the intermolecular interaction potential were used: $\sigma=0.2968 \mathrm{~nm}, \varepsilon / k=33.3 \mathrm{~K}$ for $\mathrm{H}_{2}, \sigma=0.3343 \mathrm{~nm}$, $\varepsilon / k=2703.6 \mathrm{~K}$ for $\mathrm{U}, \sigma=0.246 \mathrm{~nm}, \varepsilon / k=1040 \mathrm{~K}$ for $\mathrm{Zn}[43,44]$. The transport coefficients are calculated using fluctuation-dissipation theorems (4) generalized for binary mixer.

First consider the viscosity of the gas nanosuspension $\mathrm{U}-\mathrm{H}_{2}$. The size of the particles is equal to $1 \mathrm{~nm}$. The dependence of the viscosity coefficients of this gas nanosuspension on the nanoparticle volume concentration $\phi, \%$ at different temperatures is presented in Fig. 2. Here, the different lines correspond to the kinetic theory data obtained in [43] at 200, 300 and $500 \mathrm{~K}$ bottom-up respectively. In all cases, the volume concentration of the particle is very small (from 0.02 to $0.08 \%$ ), however the mass concentration is sufficiently large. In Fig. 2 different markers correspond to the simulation data. These data are in good agreement with the kinetic theory ones. The viscosity of gas nanosuspension increases with increasing particle concentration and it is more than the viscosity of carrier gas. The viscosity of gas nanosuspension exceeds the viscosity of the carrier gas by about $90 \%$ at concentrations of the order of $0.02 \%$ and at room temperature. This effect increases with increasing temperature. For example, at $1000 \mathrm{~K}$, the relative viscosity coefficient $\eta_{r}=\eta / \eta_{g} \approx 2.3$ (here, $\eta$ and $\eta_{g}$ are the viscosity coefficients of the gas nanosuspension and carrier gas respectively).

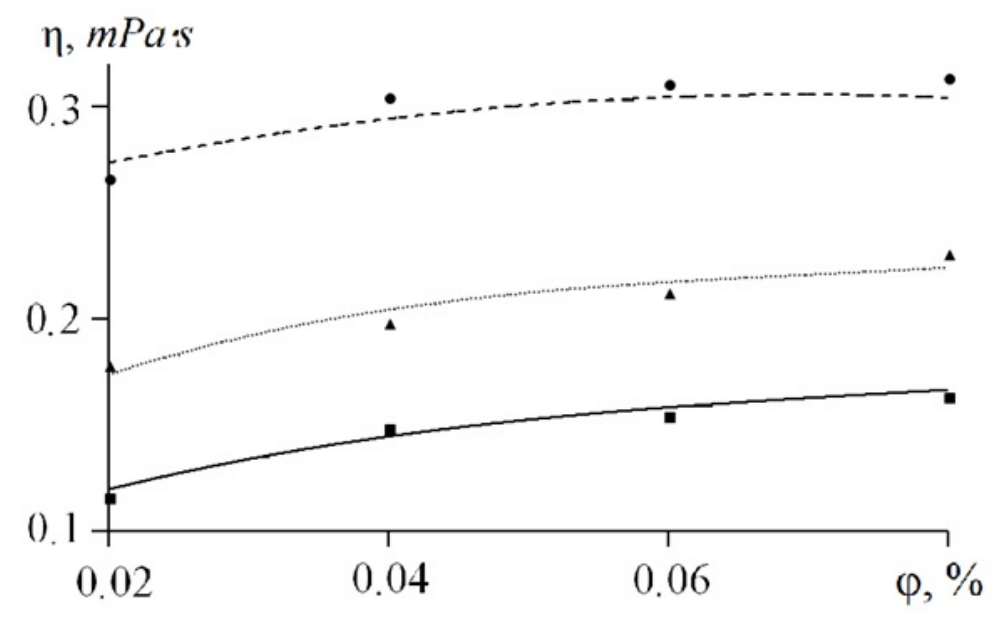

FIG. 2. The viscosity coefficients of $\mathrm{U}-\mathrm{H}_{2}$ gas nanosuspension versus nanoparticles volume concentration $\phi, \%$ at different temperature. The different markers correspond to the simulation data at the temperature 200, 300 and $500 \mathrm{~K}$ bottom-up respectively. Different lines correspond to data of the kinetic theory [43]

On the other hand, in [44], it is shown that in certain situations, gas nanosuspensions may have viscosity less than in carrier gas. To check this conclusion, the viscosity of the $\mathrm{Zn}-\mathrm{Ne}$ gas nanosuspension was calculated. The size of the particles is equal to $1 \mathrm{~nm}$ again. The obtained data are presented in Fig. 3. The lines correspond to the kinetic theory data [44] at the temperature 200, 300, 500 and $1000 \mathrm{~K}$, bottom-up respectively. The different labels correspond to our calculated data for appropriate temperature and particle volume concentrations.

The viscosity of the gas nanosuspensions considered at all temperatures is lower than the viscosity of the carrier gas. At room temperature and particle volume concentration of $0.02 \%$, the effective viscosity of this gas nanosuspension is about $15 \%$ lower than that of pure neon, and this effect increases with increasing temperature. The simulation and kinetic theory data coincide with each other. Thus the proposed algorithm permits one to model the viscosity of the gas nanosuspensions. 


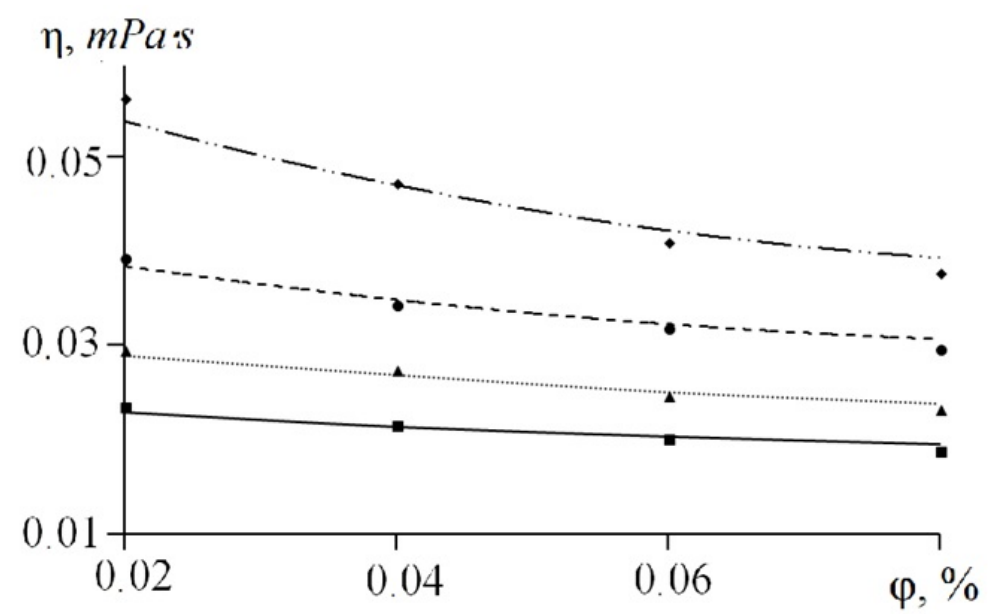

FIG. 3. The viscosity coefficients of Ne-Zn gas nanosuspension versus nanoparticles volume concentration $\phi, \%$ at different temperatures. The different labels on the lines corresponds to the simulation data at the temperature $200,300,500$ and $1000 \mathrm{~K}$, bottom-up respectively. The lines correspond to the data of the kinetic theory [44] for appropriate temperature and the particles volume concentrations

\section{Conclusion}

The proposed algorithms for calculating gas transport coefficients allow one, using a relatively small number of molecules, to obtain data comparable in accuracy with experimental results. It is the stochastic algorithm because all phase trajectories of the modelled system are built stochastically. However it is not the Monte-Carlo type algorithm, because we do not build the stochastic process to solve any equation. The dynamics of the molecular gas in corresponding phase space are modeled.

In this paper, the simulation of the transport coefficients of the rarefied gases are considered. All transport coefficients of such gases are dependent on the velocity of the particles of the system (see, for example (3) and (4)). Therefore the dynamics of the molecules in the velocity space were studied. However, strictly speaking the determination of the velocities of the particles after collision (see (2) requires knowledge of the vector $\mathbf{e}_{i j}=\left(\mathbf{r}_{j}-\mathbf{r}_{i}\right) /\left|\mathbf{r}_{j}-\mathbf{r}_{i}\right|$. There are two different ways to determine this vector during each time step. In the first case, at time $t$, all positions of the molecules are defined $\left(\mathbf{r}_{1}, \mathbf{r}_{2}, \ldots, \mathbf{r}_{N}\right)$. Then. at selected time interval $\tau_{1}$, the coordinate of the molecule $i$ is changed as follows $\mathbf{r}_{i}\left(t+\tau_{1}\right)=\mathbf{r}_{1}(t)+\mathbf{v}_{1}(t) \tau_{1}$. In this case, the calculation result is the full set of the coordinates and velocities of all the molecules of the simulated system at successive times. In the second way to determine the vectors $\mathbf{e}_{i j}$ the initial data of the molecules were used. It is clear that the second way is much more efficient. Both described procedures were used in the developed algorithm. It was shown that the accuracy of the simulation is practically identical.

In all cases, the accuracy of the simulation increases with both the number of particles used and the number of phase trajectories for averaging the results. The relative error of the viscosity coeficient calculation is well described by the following formula: $\Delta \sim 1 / \sqrt{N L}$. This means, in particular, that in order to achieve a given accuracy, the number of molecules can be "exchanged" for the number of phase trajectories. It is very important for example to model the gas nanosuspensions with large particles.

It is very interesting that the viscosity of gas nanosuspensions may be either larger or less than viscosity of the carrier gas. The decrease in the viscosity can be quite significant, at normal temperatures it can reach $10-15 \%$. This effect increases with increasing the temperature. It should be emphasized that the volume concentrations of nanoparticles are very low and do not exceed $0.02 \%$. A decrease in the effective viscosity coefficient compared to the corresponding value for the carrier gas should be expected if the sizes of the carrier gas molecules and nanoparticles differ significantly. If the sizes of the nanoparticles are sufficiently small, then a decrease in the effective viscosity coefficient can be expected if the ratio of the density of the nanoparticle material to the density of the carrier gas molecules is small enough also, e.g., when light particles are added to a heavy gas. Physically, the decreasing the effective viscosity of gas nanosuspension is due to a decrease in the frequency of molecule-molecule collisions due to collisions of molecules with nanoparticles, as well as due to a decrease in the molar fraction of gas molecules.

This work is partially supported by the Russian Foundation for Basic Research (grant No. 20-01-00041). 


\section{References}

[1] Chapman S., Cowling T.G. The Mathematical Theory of Non-Uniform Gases, Cambridge University Press, Cambridge, 1990,423 p.

[2] Hirschfelder J.O., Curtiss Ch.F., Bird R. B. Molecular Theory of Gases and Liquids, John Wiley and Sons, Inc. Chapman and Hall, Lim., 1954, 1219 p.

[3] Ferziger J., Kaper H. Mathematical Theory of Transport Processes in Gases, North-Holland Publishing Company, Amsterdam-London, 1972, 546 p.

[4] Rudyak V.Ya. Statistical Aerohydromechanics of Homogeneous and Heterogeneous Media. Vol. 2. Hydromechanics, NSUACE, Novosibirsk, $2005,468 \mathrm{p}$

[5] Norman G.E., Stegailov V.V. The stochastic properties of a molecular dynamical Lennard-Jones system in equilibrium and nonequilibrium states. J. Exp. Theor. Phys., 2001, 92, P. 879-886.

[6] Norman G.E., Stegailov V.V. Stochastic and dynamic properties of molecular dynamics systems: simple liquids, plasma and electrolytes, polymers. Comput. Phys. Commun., 2002, 147, P. 678-683.

[7] Rudyak V.Ya., Lezhnev E.V. Stochastic method of the simulation of the rarefied gas transport coefficients. Math. Mod., 2017, 29, P. 113-122.

[8] Rudyak V.Ya., Lezhnev E.V. Stochastic algorithm for simulating gas transport coefficients. J. Computational Physics, 2018,355 , P. 95-103.

[9] Klimontovich Y. Kinetic Theory of Non-Ideal Gases and Non-Ideal Plasmas, Pergamon Press, Oxford, 1982,314 p.

[10] Rudyak V.Ya. Statistical Theory of Dissipative Processes in Gases and Liquids, Science, Novosibirsk, 1987,287 p.

[11] Zubarev D.N. Nonequilibrium Statistical Thermodynamics, Consultants Bureau, New York, 1974, 371 p.

[12] Hoover W.G. Computational Statistical Mechanics, Elsevier, Amsterdam, 1991, 324 p.

[13] Ernst M.H. Formal theory of transport coefficients to general order in the density. Physica, 1966, 32, P. $209-243$.

[14] Khon'kin A.D. Equations for space-time and time correlation functions and proof of the equivalence of results of the Chapman-Enskog and time correlation methods. Theoretical and Mathematical Phys., 1970, 5, P. 1029-1037.

[15] Krishna R., Wesselingh J.A. The Maxwell-Stefan approach to mass transfer. Chemical Engineering Science, $1997, \mathbf{5 2}, 1997$, P. 861-911.

[16] Rudyak V.Ya., Belkin A.A., Ivanov D.A., Egorov V.V. The simulation of transport processes using the method of molecular dynamics. Selfdiffusion coefficient. High Temperature, 2008, 46(1), P. 30-39.

[17] Van Heijningen R.J.J., Harpe J.P., Beenakker J.M. Determination of the diffusion coefficients of binary mixtures of the noble gases as a function of temperature and concentration. Physica, 1968, 38, P. 1-34.

[18] Valander S.V., Nagnibeda E.A., Ridalevskaya M.A. Some Questions of the Kineticic Theory of Chemical Reacting Gas Mixure, LSU, Leningrad, 1977, $289 \mathrm{p}$.

[19] Zhdanov V.M., Alievskii M.Ya. Transport Processes and Relaxation in Molecular Gases, Science, Moscow, 1989,335 p.

[20] Grigoriev I.S., Meilihova E.Z. Physical values. Handbook, Energoatomizdat, Moscow, 1991, 978 p.

[21] Helfand E. Theory of molecular friction. Phys. Fluids, 1961, 4, P. 681-691.

[22] Suddaby A., Gray P. Relation between the friction constant and the force correlation integral in Brownian movement theory. Proc. Phys. Soc., 1960, 75, P. 109-118.

[23] Rudyak V. Basic kinetic equation of a rarefied gas. Fluid Dynamics, 1989, 24, 954-959.

[24] Rudyak V. Correlations in a finite number of particles system simulating a rarefied gas. Fluid Dynamics, 1991, 26, P. 909-914.

[25] Bird G., Molecular Gas Dynamics, Clarendon Press, 1976, Oxford. 302 p.

[26] Gimelshtein S.F., Rudyak V.Ya. Simulation of rarefied gas by the small number particles system. Sov. Tech.-Phys. Letters, 1991, 17, P. 74-77.

[27] Jabbari F., Rajabpour A., Saedodin S. Thermal conductivity and viscosity of nanofluids: a review of recent molecular dynamics studies. Chemical Engineering Sci, 2017, 17, P. 67-81.

[28] Rudyak V.Ya., Krasnolutskii S.L. Diffusion of nanoparticles in a rarefied gas. Technical Physics, 2002, 47, P. 807-813.

[29] Rudyak V.Ya., Krasnolutskii S.L. Kinetic description of nanoparticle diffusion in rarefied gas. Doklady Physics, 2001, 46, P. 897-899.

[30] Rudyak V.Ya., Krasnolutskii S.L., Nasibulin A.G., Kauppinen E.I. Methods of measuring the diffusion coefficient and sizes of nanoparticles in rarefied gas. Doklady Physics 2002, 47, P. 758-761.

[31] Rudyak V.Ya., Krasnolutskii S.L., Ivanov D.A. The interaction potential of nanoparticles Doklady Physics, 2012,57, P. 3335.

[32] Wang X-Q., Mujumdar A.S. A review on nanofluids - Part II: experiments and applications. Brazilian J. Chem. Eng., 2008, 25(4), P. 631-648.

[33] Wong K.V., De Leon O. Applications of nanofluids: Current and future. Advances in Mech. Eng., 2010, 2 , Article ID 519659.

[34] Usoltseva L.O., Volkov D.S., Avramenko N.V., Korobov M.V., Proskurnin M.A. Nanodiamond aqueous dispersions as potential nanofluids: the determination of properties by thermal lensing and other techniques. Nanosystems: Physics, Chemistry, Mathematics, 2018, 9(1), P. 17-20.

[35] Lari H., Morsali A., Heravi M.M. The prediction of $\mathrm{COOH}$ functionalized carbon nanotube application in melphalan drug delivery. Nanosystems: Physics, Chemistry, Mathematics, 2019, 10(4), P. 438-446.

[36] Einstein A. Eine neue Bestimmung der Molekiildimensionen. Annalen der Physik, 1906, 19, P. 289-306.

[37] Batchelor G.K. The effect of Brownian motion on the bulk stress in a suspension of spherical particles. J. Fluid Mech. 1977, 83, P. 97-117.

[38] Acrivos A. Chang E.Y. A model for estimating transport quantities in two-phase materials Phys. Fluids, 29 P. $459-464$.

[39] Mooney M. The viscosity of a concentrated suspension of spherical particles J. Colloid Sci, 1951, 6-8, P. 162170.

[40] Frankel N.A., Acrivos A. On the viscosity of a concentrated suspension of solid spheres Chem. Eng. Sci, 1967,22, P. 847853.

[41] Rudyak V.Ya., Minakov A.V. Thermophysical properties of nanofluids Eur. Phys. J. E, 2018, 41, P. 15.

[42] Rudyak V.Ya. Thermophysical characteristics of nanofluids and transport process mechanisms. J. of Nanofluids, 2019, 8(1), P. 1-16.

[43] Rudyak V.Ya., Krasnolutskii S.L. About viscosity of rarefied gas suspensions with nanoparticles. Doklady Physics, 2003, 48, P. 583-586.

[44] Rudyak V.Ya., Krasnolutskii S.L. Effective viscosity coefficient for rarefied nano gas suspensions. Atmosphere and Ocean Optics, 2004, 17, P. $468-475$. 